\title{
HIV and AIDS Programmes in Zimbabwe: Implications for the Health System
}

\author{
Tafadzwa Chevo and Sandra Bhatasara \\ Sociology Department, University of Zimbabwe, Harare, Zimbabwe \\ Correspondence should be addressed to Tafadzwa Chevo, tafadzwachevo@hotmail.com
}

Received 18 September 2011; Accepted 25 October 2011

Academic Editor: I. Mezzaroma

Copyright ( $) 2012$ T. Chevo and S. Bhatasara. This is an open access article distributed under the Creative Commons Attribution License, which permits unrestricted use, distribution, and reproduction in any medium, provided the original work is properly cited.

This paper analyzes the implications of HIV and AIDS prevention, treatment, and care programmes on the health system in Zimbabwe. The programmes have been spearheaded by various stakeholders that include the public and private sectors, nongovernmental organizations, formal and informal institutions, and intergovernmental organizations. There has been a tremendous increase of the programmes as they adapt to local contexts, accommodate new funders, and changes in population attitudes, and expectations in the country. Through a comprehensive literature review, this paper focuses on Behaviour Change, the Antiretroviral Therapy, Home-Based Care, Prevention to Mother To Child Transmission and Voluntary Counselling and Testing programmes and services in relation to the components of the health system that include health service delivery, human resources, finance, leadership and governance, and the medical products and technologies. Thus far, the implications are uneven throughout the health system and there is need to integrate the HIV and AIDS programmes within the health system in order to achieve positive heath outcomes.

\section{Introduction}

This paper analyzes the implications of HIV and AIDS prevention, treatment, and care programmes on the health system in Zimbabwe. HIV and AIDS programs have evolved tremendously since their inception, adapting to local context, entry of new funders, and changes in population attitudes and expectations in the country. These programmes have been implemented to address the multidimensional impacts of HIV and AIDS in the country. These interventions have also been spearheaded by various stakeholders that include the public and private sectors, nongovernmental organizations, formal and informal institutions, and Intergovernmental organizations such as UNAIDS, PEPFAR, UNICEF, and WHO. These programmes are a fact on the ground; they keep millions of people alive, enabling people living with HIV and AIDS to survive and sustain their families. The programs provide valued services to populations and they have become integral components of health care provision in low income and even middle income countries. Moreover, AIDS treatment is here to stay; antiretroviral treatment is for life and cannot be ethically withdrawn, implying a longterm commitment from funders. More so, in the context of existing inequities and inequalities that undermine access to care in a country with the majority of the population being poor, HIV and AIDS programmes should at least neither worsen or reproduce inequities and inequalities nor exacerbate health system human resources losses. Therefore, there is an urgent need for evidence on the implications of HIV and AIDS programmes and other categorical programming for health system strengthening.

In addition, global and national policymakers recognize that gains in HIV and child, maternal, and other health goals all require a resilient health system. This convergence between HIV programs and health systems is articulated in new aid policies, including the Global Health Initiative. Essentially, HIV and AIDS programmes produce both systems and health outcomes and there is serious concern that maintaining large HIV programs may hinder countries' ability to respond to other high priority health needs. The resulting polarization between HIV and AIDS programmes, on one hand and other disease-specific programs on the 
other hand, and efforts to build up health systems substantially warrant research on the implications of HIV and AIDS programmes on the health system in Zimbabwe.

In light of the above, there is a strong case for a systematic inquiry on implications of HIV and AIDS prevention, treatment and care programmes on the health system in order to establish how the health system can be strengthened in a resource-constrained setting like Zimbabwe. However, this paper acknowledges that providing answers to these research problems with confidence is hindered by the vast heterogeneity of HIV programs, the lack of data on non-HIV services, and the scarcity of longitudinal data on population health outcomes in Zimbabwe.

\section{Research Questions}

Main research question: what are the implications of HIV and AIDS prevention, treatment, and care programmes on the Health system in Zimbabwe?

\subsection{Subresearch Questions}

(a) In what ways have health services been affected?

(b) What are the different ways in which the human resources component has been affected?

(c) How has the health information system been influenced by the HIV programs in Zimbabwe?

(d) What has been the effect of HIV programs on health financing in Zimbabwe?

(e) What have been the effects on medical products, vaccines, and technologies?

(f) How have HIV programs influenced health governance in Zimbabwe?

(g) To what extent can HIV programs be integrated into resource-constrained countries like Zimbabwe?

\section{Methodology}

This paper is largely based on literature review. The review of the literature did not follow a strict exclusion and inclusion criteria; thus literature that contains relevant information on the theme this paper is focusing on was included. A rigorous desk review was conducted to identify the relevant literature on the impact of HIV and AIDS programmes on the health systems in Sub-Saharan Africa, Southern Africa, and in Zimbabwe in particular. From this initial review, themes on HIV and AIDS programmes and components of the health system in the country were identified.

We searched for evidence globally, however, with a specific focus on information on Southern Africa and Zimbabwe. The starting point was documents from SubSaharan Africa and Southern Africa. We critically examined the countries' HIV and AIDS prevention, treatment, and care programmes as well as those by other stakeholders such as the UNAIDS, PEPFAR, and WHO. For each programme we reviewed published, peer-reviewed literature reports, monographs, and other "grey" literature. The main sources of data were internet searches of published and grey literature from a wide range of databases, including UN agencies such WHO, UNAIDS, PubMed, POPLINE, AEGiS (AIDS Education Global Information System), Science Direct, Cab direct, and Informit Health Collection. Further information was obtained through search engines such as Google Scholar.

Essentially, snowballing technique was used to widen the search for evidence. The search strategy was such that various phrases and combination of terms were used in searching such as "HIV and AIDS", "HIV and AIDS programmes," and "Impacts of HIV and AIDS". Furthermore, on databases that can process longer phrases such as "HIV and AIDS programmes and effects on health systems" were applied.

We systematically scanned the titles, summary, executive summary, and abstract for relevance. Relevant documents were then analysed further, while those of questionable relevance were reviewed referred to colleagues for clarification. We used discourse analysis method to analyze the content of the articles. We relied mainly on electronic documents, publishers, and reference sources such as Elsevier-Science Direct, Springer Link, Wiley Online, and BMJ. Additionally we also conducted reviews of key institutions working on health systems and HIV and AIDS (such as UNAIDS and WHO) and reviewed the United Nations General Assembly Special Session (UNGASS), Ministry of Health and Child Welfare (MoHCW), and National AIDS Council (NAC) reports and policies.

We synthesised the evidence according to types of HIV and AIDS prevention, treatment, and care programmes, implications of HIV and AIDS programmes for the health system and health systems strengthening strategies. We acknowledge that our synthesis and analysis was hindered by the vast heterogeneity of HIV and AIDS programs in Zimbabwe, the lack of data on HIV and AIDS and non-HIV services in the country, and the absence of longitudinal data on population health outcomes in Zimbabwe. We were also heavily dependent on available electronic documents such that we had limited cover of literature that is not based on the internet. We also acknowledge the fact that most of the documents we obtained did not address specific issues raised by our research questions particularly relating to Zimbabwe.

\section{The Health Systems Context in Zimbabwe: An Overview}

The Zimbabwean health system has been in perpetual decline for more than a decade and the result is a systematic decrease in coverage of most basic services and a rising maternal and child mortality rate. This decline is most evident in key areas such as maternal and child health, for example, the Expanded Support Programme (ESP) on immunization and obstetric care for pregnant women, once high-performing core elements of Zimbabwe's Primary Health Care (PHC) system. In addition, even in subsectors such as access to antiretroviral treatment for HIV, where overall progress has been relatively solid, coverage for critical groups such as pregnant women or children is either unknown or very low. As a result, the disparities between urban and rural access continue to grow [1]. Based on data from the 
Zimbabwe Demographic Health Survey (ZDHS) (2005/6), Multiple Indicator Monitoring Survey (2009), Maternal and Perinatal Mortality study and other studies, the NHS (2010) has identified that Zimbabweans are dying from easily preventable and treatable conditions for example, malaria, pregnancy related complications, and diarrhoeal diseases to mention a few. Ultimately, most health indicators have either stagnated or deteriorated or both. As a result, the country is off track in most of its health targets including the targets such as the MDG target of prevalence rate of $9 \%$ in adults between 15 and 49, [1]. The health system, which is supposed to assist in improving these health indicators, has virtually collapsed.

According to the MoHCW (2010) [1], the major challenge facing the health sector is lack of resources financial, human, and material. MoHCW, 2010 [1] noted that if the current funding levels and weak capacity of the public health system persist or deteriorate, Zimbabwe will not achieve health-related MDGs. Therefore, under the Health Sector Investment Case 2010-2012, the major thrust is to revitalize the health sector, identify high impact priority interventions and mobilize additional resources to scale up progress towards attainment of MDGs, which at the moment are lagging behind and, it is anticipated that the benefits of scaling up MDG-related interventions should positively impact on the wider health system in the country.

The MoHCW is also focusing on revitalizing the PHC approach to address the diverse health needs of the nation. The same was reinforced in the Assessment of Primary Care in Zimbabwe (2009) which clearly articulated the need to "put in place a backed by clear service entitlements, with resources effectively applied to community and primary care levels of the health system" as "an entry point to wider PHCoriented changes" [1].

In addition, in line with the PHC approach, which calls for a conscious acceptance by the community of the responsibility for its own health, the tradition of community involvement in health has been sustained in the country. A number of home- or community-based health practices or behaviours that can be carried out by households or communities themselves after receiving guidance are also outlined. Thus, the role of the health system, in this context, is to empower communities through information, education, and other communication strategies, as well as other forms of support, for example, in the form of provision of insecticidetreated mosquito nets, provision of safe drinking water, and sanitation and provision of oral rehydration sachets among others.

Community health workers are also the key link between communities, especially rural, and local health services. They mobilize households and communities in activities that foster promotive, educative, and preventive health behaviour. In Zimbabwe, Village Health Workers (VHWs) are the commonest community health workers in rural communities where they provide services in the prevention of locally endemic conditions, treatment of simple conditions disease, and surveillance. Rural health centres also provide appropriate medicines and commodities and provide ongoing technical supervision and support to VHWs. Ideally one village health worker should serve 100 households or a village [1]. The equivalents of VHWs in urban areas are called Health Promoters. Community-Based Distributor whose main function is to promote family planning services including the resupply of appropriate contraceptives, is another key community health worker. A growing range of community health workers includes former chloroquine holders, depot holders, and home-based caregivers [1].

It is also imperative note that the Government of Zimbabwe has continued to demonstrate commitment to the health of the nation by its national response to HIV and AIDS. The country NAC which was established by an Act of Parliament in 1999 and has a broad-based mandate to provide for measures to combat the spread of HIV. In 2008, changes were made to the NAC Board to include representation from labour, business sector, and People Living with HIV and AIDS and increase representation of women from two to three. This has enabled the national council to integrate diverse health needs relating to HIV and AIDS in its mandate.

\section{Conceptual Framework}

The framework used in this paper is borrowed from the WHO (2007) understanding of a health system. It defines a health system as all organizations, people and actions whose primary intent is to promote restore or maintain health. The framework highlights six health system components including the description of six health system building blocks which are (a) health services, (b) health work force, (c) health information system, (d) medical products, vaccines, and technologies, (e) health-financing system, and (f) leadership and governance [2]. This paper in analyzing the implications of HIV and AIDS prevention, treatment, and care programmes on the health system in Zimbabwe used the following conceptual framework in Figure 1.

\section{HIV and AIDS Programmes in Zimbabwe: A Review}

Zimbabwe is one of the 189 countries that have committed themselves to a comprehensive programme of national commitment and action to fight the HIV and AIDS epidemic by adopting the United Nations General Assembly Special Session (UNGASS) declaration of commitment on HIV and AIDS of June 2001. The declaration of commitment established a number of goals for the achievement of specific quantifiable and time-bound targets, including reductions in HIV infection among infants and young adults; improvement in HIV and AIDS education; health care and treatment; improvement in orphan support. The following is a review of some of the HIV and AIDS prevention, treatment, and care programmes.

6.1. Prevention of Mother-to-Child Transmission Programmes. Kak et al. (2011) observed that each year, over half a million newborns are infected with HIV in sub-Saharan Africa through mother-to-child transmission and HIV infection 


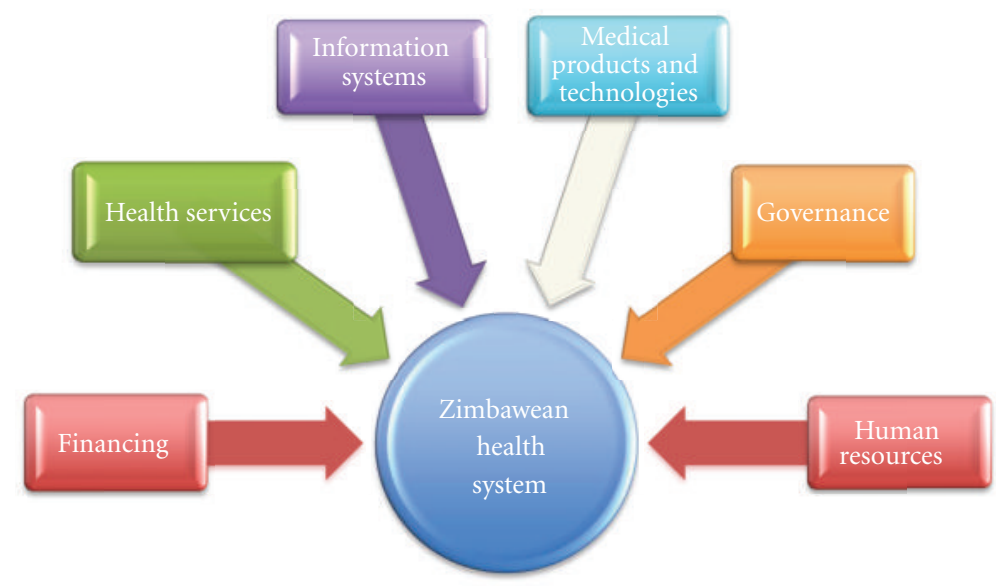

Figure 1: Zimbabwean health system.

rates among pregnant women range from 15 to 40 percent in the countries with the highest overall HIV prevalence, with women of reproductive age comprising over 55 percent of HIV-infected adults [3]. It is understood that the impact of HIV may contribute up to $18 \%$ of maternal deaths, through increased risk of malaria, tuberculosis, and other infectious diseases for HIV-infected mothers. Furthermore, HIV has an extended effect, impacting maternal mortality over the first 24 months after delivery. The link between maternal HIV and the health of the infant has been demonstrated such that infant mortality rates are 4 times higher in infants whose mothers died of HIV than those whose mothers were alive. It is documented that paediatric HIV infection results in 210000 child deaths each year in sub-Saharan Africa, 4\% of all child deaths [3]. In high HIV-prevalence countries, a substantially higher proportion of deaths are attributable to paediatric HIV. In light of this, PMTCT programmes have taken a centre stage in many countries including Zimbabwe.

A comprehensive PMTCT approach includes the following four components that address Maternal, Newborn, and Child Health throughout the continuum of care; preventing $\mathrm{HIV}$ infection in women of reproductive age; avoiding unintended pregnancy among HIV-infected women; preventing transmission of HIV from an HIV-infected woman to her infant during pregnancy, labour, childbirth, and breastfeeding through HCT, ARV drugs for prophylaxis, ART for those who are eligible, safer infant feeding practices, and providing followup and care, support and treatment to HIVinfected women, their infants, and families; ART using the new WHO PMTCT guidelines for ARV drug prophylaxis which recommend giving HIV-infected pregnant women the drug Azidothymidine (AZT) from 28 weeks, combined with single-dose Nevirapine at birth to the mother and infant [3]. Ideally, mothers should receive one week of further treatment of AZT and 3TC postnatally in order to reduce the likelihood of developing viral resistance to Nevirapine. These regimens, although very effective in clinical trials, have not been implemented at scale in high HIV prevalence settings [3].

The argument with the PMTCT programme in Zimbabwe is that it has achieved commendable success and visible failures yet the implications on the health system have not been captured in mainstream policy and academic discourses. It is imperative to note that prevention of motherto-child transmission of HIV (PMTCT) has been one of the strongest pillars of the HIV and AIDS responses in Zimbabwe. According to the MoHCW, PMTCT is integrated within the broader framework of reproductive health service provision [4]. The country has demonstrated immense commitment in developing and utilising policy guidelines on the expansion of the PMTCT programme. To acknowledge also is that a multisectoral national PMTCT partnership forum was established to improve coordination of the programme. As noted by the MoHCW, the existing national PMTCT protocols were amended in 2008 in line with the WHO recommendations (revised in 2006) [5]. The country's commitment is shown in that the revised treatment guidelines were distributed to all Provincial Medical Directors, City Health Directors, Zimbabwe Association of Church related Hospitals, Zimbabwe Nurses Association, Zimbabwe Medical Association, and other relevant implementing partners. The MoHCW observed that as a result of the efforts made to expand the PMTCT programme, the country has seen an increase in uptake of PMTCT by pregnant women [6]. Figure 2 below shows the comparison of the PMTCT programme performance over 5 years in the country, from 2004 to 2008 .

According to the UNGASS, until recently the comprehensive PMTCT services in Zimbabwe were based on the single-dose Nevirapine to reduce mother-to-child transmission [7]. The MoHCW noted that by December 2008 the country had started rolling out a multiple-dose PMTCT regimen [5]. At the same time, comprehensive PMTCT sites increased from 710 in December 2007 to 920 in December 2008 and 960 in 2009. Consequently, PMTCT coverage increased from $22 \%$ in 2007 to $42.6 \%$ in 2008 [7]. The MoHCW further highlighted that early HIV infant diagnosis was introduced in 2008 using the HIV DNA PCR testing at the National Medical Reference Laboratory [5].

In 2008, 76 Primary Care Counsellors were trained on PMTCT, counselling and infant feeding [7]. It is understood that more needs to be done to train primary counsellors 




Figure 2: Data from the National PMTCT Programme database (estimated to be $80 \%$ complete from National Blood Services Zimbabwe, Annual Report, 2008).

and cadres certified to perform rapid testing to strengthen the PMTCT program. In promoting optimal and safer infant feeding practices, 6,797 infants exposed to HIV were provided with alternative feeding in 2008 [7]. The MoHCW Nutrition Department and Zvitambo conducted campaigns on exclusive breast feeding to encourage both HIV-positive and -negative women to stick to this practice. The PMTCT and reproductive health departments have been working in collaboration with PPF partners to revise the antenatal card to include information on HTC including HIV status so as to enhance tracking of children born to HIV-infected mothers. However, it is recognized that the PMTCT program besides strengthening current strategies needs to adopt, adapt, and implement the 2009 WHO rapid advice on PMTCT and feeding practices for "HIV-Exposed Infants" in the country [7].

\section{Voluntary, Counselling, and Testing Services}

The implication of the VCT programmes in Zimbabwe on the health system also present a strong case for rigorous analysis. The VCT has been identified as an entry point to most HIV and AIDS interventions globally. According to Oberzaucher and Baggaley (2002), VCT is not only a key component of both HIV prevention and care programmes but is also the gateway to both prevention and care [8]. In Zimbabwe, in 1998, counselling and testing VCT guidelines and an operating procedure manual were developed to guide implementation and in 1999, the National AIDS Policy was launched, in which protocols for rapid HIV testing were developed and, after extensive evaluation was approved for national use in 2001. Thus far, the Zimbabwe National HIV and AIDS Strategic Plan and the Health sector HIV Prevention Strategic Framework have identified HCT as an important component of the national response. The Zimbabwe National HIV Testing and Counselling Strategic
Plan 2008-2010 was launched in 2008 and broad objectives of this strategic plan emphasize the need to increase the percentage of Zimbabwean population that knows its HIV status, from $20 \%$ to $85 \%$ by 2010 and to expand services using PITC and Client-Initiated Counselling and Testing, formerly VCT [9].

The VCT has also been recognized by various stakeholders such as UNAIDS as the entry point in HIV and AIDS prevention and as

(i) a public health and developmental priority,

(ii) a human rights imperative,

(iii) a cost-effective preventive measure, particularly in high-prevalence communities,

(iv) a central to interventions such as PMTCT, access to care and support, and injecting-drug-use harm reduction,

(v) a way to provide individuals with an opportunity to plan for the future and gain access to appropriate health and support services,

(vi) a means to destigmatize and normalize HIV and to empower HIV-positive people in the community,

(vii) a mechanism that enhances the capacity of health systems to deliver appropriate services (as shown in Figure 3 below).

As presented by the UNGASS in the ZDHS of 2005/2006, $5.9 \%$ women and men (15-49 years) had been tested and received their HIV results in the 12 months prior to the survey [7]. In addition, the country had set a target to increase the percentage of people who know their status from $20 \%$ in 2007 to $85 \%$ by 2010 in line with MDGs targets. Important to note on VCT in Zimbabwe is also that a variety of HCT materials and guidelines were developed between 2008 and 2009 and, these; include the national HCT training manual for health workers; Zimbabwe National Guidelines for HCT in Children (2008); a training course for counsellors on HCT for Children (2008); PICT and HIV counselling and testing training manuals [7].

It is also important to highlight that in an effort to scale up VCT services the MoHCW adopted a number of delivery mechanisms which are integrated model within the public health institutions; stand alone model manned by NGOs; private sector workplace model; mobile outreach services conducted by NGOs: PICT model [7].

In practice, geographic coverage of testing and counselling services expanded to reach all populations during the period 2006-2007 and the number of stand-alone and integrated testing and counselling sites increased from 547 sites in 2006 to 649 at the end of 2007 [7]. HCT services were decentralized and expanded through the PMTCT program in which more than 920 health facilities were actively providing HCT services by the end of 2008. The number of clinics registered to provide testing and counselling in combination with mobile service delivery increased over the year [9]. Estimates in terms of centers providing HCT by the end of 2009 were as follows: 27 VCT centres managed 


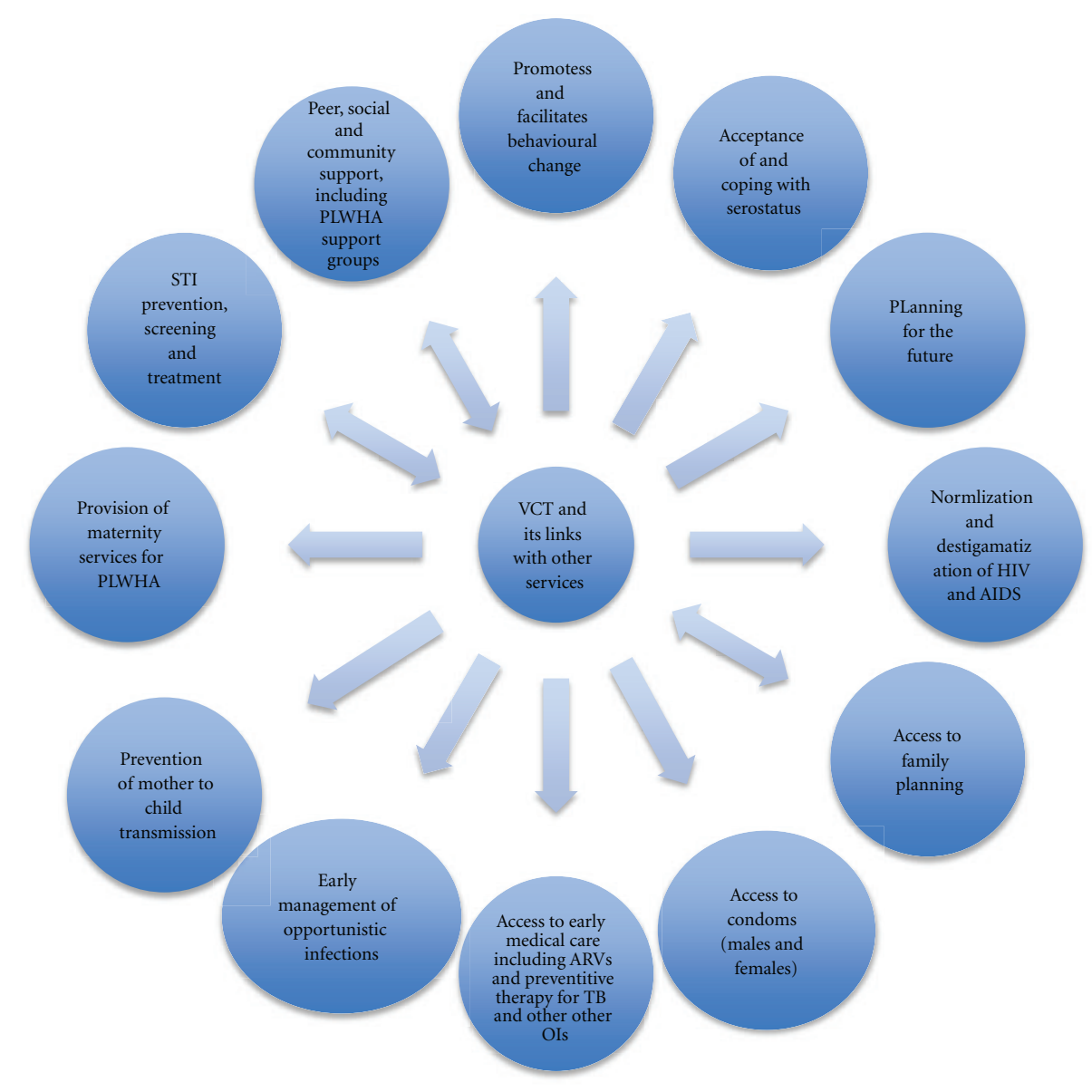

Figure 3: Source: VCT, UNAIDS technical update May 2000.

by NGOs, 502 HCT service delivery points integrated with health services, and approximately 1,000 counselling and referral-only service delivery points. Besides HTC, clients receive information on behaviour change, referrals for care, treatment and psychosocial support as well. Comprehensive and quality care is guaranteed through a strong referral system for other medical conditions such as STI, TB, and family planning [7].

\section{National Behaviour Change Programme}

The national behaviour change programme in Zimbabwe has the primary objective of reducing sexual transmission of HIV by addressing key drivers such as multiple concurrent sexual part, age different sexual relationships, and long-term discordant couple. The NAC carries out its programme in 16 districts supported by the ESP with an additional 10 districts supported by the European Union. Projects focus on community assessment, working with community leaders and facilitating community dialogue on issues in relation to culture and behaviour change.

The NAC behaviour change programme also has a special focus on youths that are out of school, usually referred to as the "not in education or training." The programme on youth out of school and training has culminated into youth friendly centres and corners providing information about HIV and AIDS. However, this programme has been facing challenges with regards to its financial sustainability [7].

In reducing sexual transmission, the NAC focuses on making available public sector and socially branded condoms to remote areas as well as urban and rural populations. Condom promotion and distribution are both a public sector MoHCW and ZNFPC and PSI project. In collaboration with its partners NAC also continues to secure genital models for condom education and demonstrations. NAC highlights that the significant challenge for this programme is increasing the staffing levels to expand condom distribution to underserved areas and new settlements such as the new fast track land resettlement areas.

Within the BC programme NAC focuses on MARPs. These constitute of the sex workers, cross-border traders, women, young people, men who have sex with men, mobile populations, truckers, internally displaced people, uniformed personnel (soldiers, police, game rangers, customs, and immigration officers), prisoners, the physically challenged, survivors of rape and sexual abuse, illegal immigrants, and injecting drug users. HIV prevention programs to this category include the distribution of male and 
female condoms and information education communication materials about HIV, stigma and discrimination, behaviour change, STIs treatment and care, and VCT.

\section{Antiretroviral Therapy Programme}

The MoHCW is responsible for the provision of ART in Zimbabwe. In December 2004 it implemented a plan that ensured the distribution of ART throughout the country. In a review commissioned by the ministry in 2007 the coverage has since increased from 5000 people to 100000 people, a $29 \%$ increase [9]. Furthermore there continues to be a concerted effort to provide Antiretroviral drugs as espoused in its plan of 2008-2012. This plan is supported by key donors and actors such as the national pharmaceutical body, NatPharm, UNICEF, United States Government, Clinton Foundation, and the NAC.

The logistical mechanisms in place to ensure the distribution of the ARVs are as follows. Once the drugs are procured and arrive in the country, NatPharm delegates the distribution of the drugs to MoHCW AIDS and TB Logistics Sub-Unit (LSU), which was set up by the JSI/DELIVER project [9]. The number of adults and children accessing ART were 148144 (39.7\%) in December 2008 and 215109 $(56.8 \%)$ in November 2009 whilst the number of children accessing ART was 8627 (24.8\%) in 2007, 13287 (38.7\%) in 2008, and $20003(57.1 \%)$ in 2009 [9].

However, funding gaps have been an obstacle in terms of realizing universal access to ART in Zimbabwe, hence; innovative mechanisms have been put in place to subsidize local manufacture of ARVs locally. One way has been to provide foreign currency for the purchase of raw materials and waiver of duty on raw materials for local production of ARVs and imported ARVs in 2008. This has improved the supply of ARVs to the extent that minimal numbers of sites have been experiencing drug stockouts.

One major concern, however, has been that the WHO recommendations that state that the CD4 $(<350)$ threshold will be the standard for commencing ART, will dramatically increase the number of people in need of ART. The Ministry states that for example in 2009, using the less than 350 CD4 criteria adult patients in need of ART increased from 317894 to 500857 [9].

\section{Home-Based Care Programme}

The government of Zimbabwe through the NAC, has come to the realization that Home-Based Care (HBC) plays a vital role in the response to HIV. This is a key strategy to an overwhelmed public health and welfare system. With the weakening economy combined with the growing number of people living with HIV and AIDS the government recognized that the health system alone could not cope with the impact of the epidemic. The NAC recognizes community homebased care as an extension of the health care delivery system that needs to be fully developed and supported as an essential component of the continuum of care for people living with HIV and AIDS and their families.
Through the NAC the community home-based care programmes prescribe a minimum package of care to highlight the value of community home-based care to the people of Zimbabwe. The programme is also in place to raise awareness about available resources and encourage support of the community and local health care providers. It provides a framework for caregivers in the implementation of CHBC activities. According to Irish AID Health Development Network and Southern Africa AIDS Information Dissemination Services (2008) most HBC programmes and services have fallen short in terms of the quality and effectiveness of their service provision. Therefore, the NAC has put in place National Community Home-Based Care Standards (2004) and the National Home-Based Care Training Manual (2005) to help standardize HBC activities, training, and other processes. Their purpose was to give programme managers and care providers a foundation from which to identify gaps in their services and to seek the training and support they needed. The introduction of ARV has also brought some new challenges to $\mathrm{HBC}$ in Zimbabwe and services. Caregivers have had to be trained on how to manage ARV therapy for people living with HIV and AIDS.

However, many $\mathrm{HBC}$ programmes and services are operating under terrific strain and hardship, with very little support. The sociopolitical and economic decline and poverty in Zimbabwe has resulted in that many funding organizations have either reduced or frozen aid to humanitarian activities in Zimbabwe. According to the Irish AID Health Development Network and SafAIDS (2008), UNICEF estimated that the average amount of international HIVrelated funding available each year in southern Africa region was US \$74 per person infected with HIV, in Zimbabwe that figure drops to US $\$ 4$. In 2007 , the figure had risen to US $\$ 17$, though still far below regional estimates. This is despite the fact that Zimbabwe has had a high rate of HIV prevalence. Reduced donor support is currently undermining the effectiveness of HBC programmes as organizations either scale down or reach out to clients with very limited services [10].

Although volunteer caregivers are keenly aware of the needs, their efforts are hampered by a lack of basic provisions: medication, gloves, soap, food, and other basic supplies. The quality of care in many $\mathrm{HBC}$ activities is severely compromised as a result of significant and unmet needs. HBC programmes are having to make do with very few resources and are in desperate need of material support.

\section{Health Systems Implications of HIV and AIDS Programmes: A Critical Analysis}

There is a strong relationship that exists between HIV and AIDS prevention, treatment, and care programmes and the health system in Zimbabwe from the literature that we reviewed. There are multidimensional positive and negative implications of HIV and AIDS programmes on the health system. However, this analysis needs to be interpreted with caution because accounts of positive and negative effects of HIV and AIDS prevention, treatment, and care programmes are available in general and specific evidence on the implications of the programmes on the health systems in Zimbabwe 
is slim. From our comprehensive literature review and analysis, many arguments suggesting the impacts of HIV and AIDS investments on health systems are based on speculation and anecdotes, small plots studies, or on early stages of the programs that cannot yet be generalized. In addition, the scaling up of programmes such as ART, provider-initiated counseling, and testing and comprehensive home-based care is fairly recent in the country. Therefore, for this paper, it would be imprudent to draw any concrete conclusions on the health system impacts of HIV and AIDS programmes in Zimbabwe.

At various levels of the health system in Zimbabwe, HIV and AIDS prevention, treatment, and care programmes produce various effects to the health systems components. For example, HIV and AIDS programmes by locating PMTCT sites in district and mission hospitals, the programme was able to rapidly scale up to 155 centres, supported by guidelines, Nurse training, manuals and protocols to standardise implementation and some facilities are using trained lay counsellors to decrease the work burden on nurses [11]. In some instances, ART introduced through Voluntary VCT services that link to PMTCT at antenatal care services, addressing female access as a point of entry, to health services particularly given that they are often excluded from mainstream health care. This has also had other positive effects by helping to deal with social stigmas around AIDS in women and providing a better opportunity for treatmentprevention links. Furthermore, El-Sadar et al. noted that ART has been shown to have indirect effects on families and communities, apart from the direct benefits to those taking the medications. Several studies have demonstrated such indirect effects of ART on child survival, in which treatment of HIV-infected mothers has reduced mortality of uninfected children, orphanhood, and the incidence of infant diarrheal disease [12]. In this regard, HIV and AIDS programmes have been positive on some aspects of the health system.

However, in relation to ART, El-Sadar et al. highlighted that the expansion of access to antiretroviral therapy for millions of persons living with HIV in low-income countries has been lauded by many yet the investment in such programs has at the same time been criticized by others, who claim diversion of resources from HIV prevention efforts and from other important health threats [12]. It is argued that the provision of ART exacerbates existing inequities and weaknesses in health systems. Additionally, some critics maintain that HIV and AIDS have received a disproportionate share of global health funding and that HIV programs were overwhelming health systems and were not sustainable.

PMTCT programmes have become imperative as a good entry point for women to learn about access to other health programmes, services, and health resources, such as support groups, sexual health, nutrition, and home-based care, and for bringing in their partners and children who may also be HIV positive. Whilst women identified as clinically eligible through PMTCT are referred to treatment programmes, those who are still relatively healthy are followed up through community outreach and advice given for child health including vaccinations and providing ongoing support for them to stay healthy.

It can be argued that the antiretroviral therapy programme is improving general health indicators such as life expectancy through increasing survival of infected individuals. The percentage of adults and children with HIV known to be on treatment 12 months after initiation of ART was $75 \%$ in the 2009 cohort analysis carried out in 22 districts that were supported by Global Fund round in Zimbabwe. The percentage of infants born to HIV-infected mothers that were infected were estimated using spectrum at $32.3 \%$ in 2007 , $31.1 \%$ in 2008 , and $30 \%$ in 2009 . The marginal decline in infection rates could be due to increase in PMTCT services uptake between 2007 and 2009 [13].

One anticipated evidence suggests that HIV and AIDS programmes expansion has to some extent increased utilization of non-HIV-related services to advance broader health goals. The impact of scaleup has not been consistent in Zimbabwe yet some programmes are contributing to the expansion of control efforts for related conditions, enhancing utilization of sexual and reproductive health services (e.g., with male circumcision), promoting access to better nutrition (in PMTCT), improving infection control in healthcare facilities (in linking Tuberculosis to HIV), promoting task shifting and health worker training, enhancing infrastructure and laboratory services, and facilitating a shift from an episodic to a continuous model of care.

At a more general level, although there are some reservations, HIV and AIDS programs have helped strengthen the national health system by attracting vital new financial resources, building systemic capacity, and introducing chronic disease management approaches time in many resource-limited settings like Zimbabwe. The rapid expansion in the number of persons receiving ART means that the health system must continue to provide acute life-saving care for those with advanced HIV/AIDS although also providing chronic care services to expanding cohorts of more stable patients who are doing well on ART [14]. This expansion has meant a transition from an emergency response to the epidemic, characterized by a public health approach, to a more integrated and durable approach to HIV and AIDS prevention, care, and treatment services. In a positive light, these have motivated a change in the health system from a fractured demoralized system barely able to cope with acute illnesses to that able to provide the continuity care necessary in the management of a chronic condition such as HIV and AIDS. The authors also highlight that innovations in governance, models of care, procurement of medications and commodities, human resource utilization, and other domain catalyzed by HIV programming can serve as a platform on which to build a response to other health threats. However, the rapid scaleup of HIV and AIDS interventions has also exposed the frailty of the health system to effectively mount an HIV response and cope with management of AIDS as a long-term illness.

Besides the fears that existing inequalities in health care may be intensified in scaling up HIV and AIDS services and programmes, there is evidence that HIV and AIDS programmes have had both negative and positive effects on 
human resources in the health sector in Zimbabwe in terms of recruitment, distribution, and retention of health care workers. Early studies reported negative effects of HIV and AIDS and the delivery of HIV and AIDS services on health care workers' morale with stigma, burn-out, resignation, and deaths due to HIV and AIDS, while more recent ones speak of hope, high prestige, high motivation, and better retention of HCWs in HIV and AIDS programmes, largely due to effective ART which has improved the prognosis of AIDS [15]. However, new cadreship has been brought in to support HIV and AIDS services, including expert patients. Innovations such as task shifting and the integrated management of adult and adolescent illness have been applied to more efficiently use available health care workers. Many vertical programmes also recruit their own health care workers, especially counsellors and home-based caregivers. This has increased the pool of health care workers and such programmes have also attracted a lot of funding, especially through Global Health Initiatives such as the Global Fund and PEPFAR.

Although some positive aspects can be seen on the human resources component of the health system, by relying on the health system for the more skilled health professionals, the programmes have also undermined other health programmes such as those for PHC. There is also evidence which suggest well-run HIV and AIDS services can serve to attract and retain health care workers as they offer incentives, such as higher salaries or salary supplements, better furnished facilities, and appointment at higher levels. However, this has had very negative effects on the health system human resources as these incentives targeted at workers for HIV and AIDS programmes exclude other health care workers. These selectively applied incentives also tend to demoralise and discourage those who are excluded.

The expansion of HIV and AIDS programmes in Zimbabwe also raise the issues of sustainability. It has been observed that the remarkable success of HIV scaleup has inevitably spawned new debates about sustainability, the need for health systems strengthening, and whether funding for HIV has been disproportionate to funding for other health needs [16]. The notion of sustainability is usually defined as the ability of country health programs to continue independent of international aid has come under increasing scrutiny in Zimbabwe. It is important to note here that Zimbabwe currently has been denied funding from the Global Fund for TB Malaria and AIDS to fund HIV and AIDS health needs yet the country has achieved a commendable reduction in HIV prevalence rate. It is also imperative to acknowledge that some widely lauded programs, such as the ESP on immunization and the polioeradication programme, have yet to achieve sustainability.

The governance component of the health system has also been transformed by HIV and AIDS programmes. As a result of these transformations there is now an unquestionable strong political awareness for health, in government and in civil society. Within the Zimbabwean health system a number of NGOs and people living with HIV and AIDS are to some extent now included in the decision-making processes through a number of coordinating mechanisms, such as the NAC. Programmes have also led to the participation of previously discriminated populations such as drug users, sex workers, and men who have sex with men. The planning, transparency of management, monitoring and evaluation, and technical assistance from external sources have resulted in sharpened and more focused decision making, thus having a positive effect on the health system.

HIV and AIDS programmes have also relatively reduced discrimination of various disarticulated social class categories such as those of HIV-positive people. For example the Zim-UNAIDS report highlights that discrimination of HIVpositive people is prohibited by Zimbabwean government under National HIV and AIDS Policy of 2000 and the Statutory Instrument (SI 202) of 1998, fundamentally the result of a number of HIV programs that target stigma [17]. One can also highlight that such instruments will have other knock on effects that will lead to reduction of stigma or discrimination because of illness. However, despite the current lack of legal frameworks to support targeting of high risk groups such as sex workers, prisoners, men who have sex with men, and IDUs with prevention activities, lobby groups representing gays and lesbians living in Zimbabwe and organisations working with sex workers are in existence and need to be formalised to strengthen the health system. Targeted programs such as condom promotion and other prevention strategies in order curb the spread of the HIV among these groups will further strengthen the health system and it is through effective governance that will ensure that these are recognized. With regards to strengthening the health system, more needs to be done to strengthen and make the health system more water tight by protecting subpopulations such as men having sex with men, intravenous drug users, and commercial sex workers.

Another major component that constitutes the health system is the financing of health care. HIV and AID programs with regard to health financing have been set up in a pragmatic way that in principle lead to a limited effect on the financing of the health system. The Zimbabwean government raises funds through the national budget and NATF. The NATF is a $3 \%$ levy collected from taxable income from all sectors to mitigate the impact of HIV and AIDS that is channelled to NAC by the Ministry of Finance. In effect every taxpayer pays for $3 \%$ of income to the NATF. The Government of Zimbabwe through the national budget contributed US \$10 596393.00 in 2007, US \$354 661 in 2008 and US \$7 491453 in 200917 towards HIV and AIDS programs [7]. The bulk of the funds are channelled to the MoHCW and the rest to other government ministries for their workplace programmes. The AIDS levy contributed US $\$ 8148$ in 2008 and US \$5 143108 in 2009 [7]. In 20082007 the AIDS levy was essentially nonexistent in 2007-2008 due to economic challenges the country was facing. Since dollarization of the economy in February 2009 monthly income stream has been improving significantly.

Still on finance, there has also been the bilateral and multilateral partners as well as international foundations that have contributed US \$35351 861.84 in 2007, US $\$ 24987127.00$ in 2008, and US \$37796697.00 in 2009 
towards HIV and AIDS programs [7]. Additional resources were made available through the Expanded Support Program, which is a grouping of the following development partners: Canadian International Development Agency, Department for International Development, Norwegian Aid, Irish Aid and Swedish International Development Agency. A total of US \$42 million was committed for the period 20072009 , of which $50 \%$ was earmarked for care and treatment [7]. Prevention accounted for $12 \%$ of the ESP budget, while $10 \%$ was allocated to monitoring and evaluation [7]. This approach added value to existing programs, by adopting community home-based care programs, TB treatment and PMTCT as entry points for ART, and encouraging PLWHA, including those on treatment, to become prevention activists. The mitigation programme for orphaned and vulnerable children received significant funding from the programme of support, a pooled donor funding setup to finance implementation of the NAP for OVC. The fund committed US \$84 million for 3-year funding for the National Action Plan (NAP) for OVC. The NAP supports OVC education, healthcare, birth registration, and access to HIV/AIDS prevention, treatment, and care and support services. Therefore, with regard to funding, HIV programmes have had a positive effect on the health system, given that additional sources of funding have been sourced from the Zimbabwean tax payer and from other development partners.

\section{Conclusion and Recommendations}

This study has demonstrated that there is a strong relationship that exists between HIV and AIDS prevention, treatment and care programmes, and the health system in Zimbabwe. From the literature reviewed, there are multidimensional positive and negative implications of the programmes on the health system. For instance, ART has reduced mortality and morbidity from HIV and AIDS while improving marginally the general life expectancy. The analysis has also revealed the need for research focusing on the implications of the HIV and AIDS programs given that the available data on effects of HIV and AIDS programs on the health system in Zimbabwe limited.

Although there is agreement that stronger health systems are needed to achieve improved outcomes in HIV and AIDS programmes, there is much less consensus on how to strengthen health systems to cope with the expanded programmes. There are a number of challenges facing the national health system in Zimbabwe including declining public health indicators, financial and human resources constraints, how to evolve the public health response to accommodate the increasing complexity of long-term HIV and AIDS prevention, treatment and care, and how to integrate HIV and AIDS services into mainstream health programs to ensure a durable response, at the same time confronting persistent health systems bottlenecks and suboptimal and inefficient service delivery designs within a constrained economic context. A number of health system strengthening actions have been used to mount a response to the HIV and AIDS programmes in various contexts. However, there is limited evidence on what works in resource-constrained countries such as Zimbabwe to deliver quality long-term HIV and AIDS prevention, treatment and care without weakening health system, and undermining HIV and AIDS programmes at the same time. In this paper, we suggest two approaches, health systems strengthening approach and services integration approach. Integration refers to a spectrum of organizational arrangements related to the funding, administration, organization, service delivery, and clinical scenarios designed to create connectivity, alignment and collaboration [18].

Ekouevi et al. argued that there is need to strengthen health systems to deliver primary health care for those with and without HIV and AIDS, and it is also essential to build and take advantage of synergies across health information systems [19]. In addition, integrating services, strategies, and plans may not only improve equity, access, and coverage, but may also enhance the quality and efficiency of health care. In this regard, to realize these synergies, HIV and AIDS programs must be implemented within a primary healthcare framework that is able to provide integrated services that address multiple patient needs across a continuum of care.

In order to prevent the weakening of the health system, it is also imperative to move away from the current situation of unplanned "spill-overs" to a more systematic and active management of the synergies between HIV/AIDS programs and health system strengthening in the country. This requires concerted efforts for a policy and technical framework, which will guide actions to avoid threats maximize the synergies between HIV/AIDS investment and health systems. However, in order for the health systems strengthening and services integration to work, there is need for comprehensive documentation of the impacts on health systems of investment in HIV and AIDS programmes. Jones and others noted that investment of a modest proportion of resources in public health leadership training programmes can assist in building capacity to lead and manage national HIV and other public health programmes [20]. This will also strengthen information management. More in-depth studies should be undertaken on the health system in the country focusing agreed-upon frameworks and measurements.

More so, there is need to strengthen HIV and AIDS service delivery and integrate it into the primary health care system. Yu and others alluded to the view that in the long run, effective prevention, treatment, and care for HIV and AIDS should be integrated with the existing health service and system because AIDS is a chronic disease and WHO has proposed a public health approach to ART to enable scaled-up access to treatment for HIV-positive people in developing countries, which entails standardized, simplified treatment protocols, and decentralized service delivery [21]. This can be a better option in Zimbabwe in the face of economic austerity. At the same time, although the country has currently been denied funds for HIV and AIDS under the Global Fund, it should continue to advocate for increased funding for primary health care which the country cannot sustain using domestic resources. 


\section{Abbreviations}

AIDS: Acquired Immune Deficiency Syndrome

ARV: Antiretroviral

ART: Antiretroviral Therapy

CBD: $\quad$ Community-Based Distributor

ESP: $\quad$ Expanded Support Programme

HBC: Home-Based Care

HCT: $\quad$ HIV Counselling and Testing

HIV: $\quad$ Human Immune Virus

MGDs: Millennium Development Goals

MoHCW: Ministry of Health and Child Welfare

NAC: National AIDS Council

NAP: $\quad$ National Action Plan

NATF: National AIDS Trust Fund

NHS: National Health Strategy

OVCs: Orphaned and Vulnerable Children

PEPFAR: The U.S. President's Emergency Plan for AIDS Relief

PHC: $\quad$ Primary Health Care

PICT: Provider Initiated Counselling and Testing

PSI: $\quad$ Population Services International

PMTCT: Prevention of Mother to Child Transmission

SAFAIDS: Southern Africa AIDS Information

Dissemination Services

STISs: Sexually Transmitted Infections

VCT: Voluntary Counselling and Testing

VHWs: Village Health Workers

UNAIDS: United Nations AIDS

UNGASS: United Nations General Assembly Special Session

UNICEF: United Nations International Children's and Educational Fund

WHO: World Health Organization

ZDHS: Zimbabwe Demographic Health Survey

ZNFPC: Zimbabwe National Family Planning Council.

\section{References}

[1] Ministy of Health and Child Welfare, The Zimbabwe Health Sector Investment Case (2010-2012): Accelerating Progress Towards the Millennium Development Goals, MoHCW, Harare, Zimbabwe, 2010.

[2] World Health Organization, Everybody's Business: Strengthening Health Systems to Improve Health Outcomes: WHO's Framework for Action 2007, 2008.

[3] Y. Kak, I. Chitsike, C. Luo, and N. Rollins, Prevention of Mother-to-Child Transmission of HIV/AIDS Programmes, Opportunities for Africa's Newborns 113, 2011.

[4] Ministry of Health and Child Welfare, PMTCT and Paediatric HIV Prevention, Treatment and Care National Plan, 20062010, 2006.

[5] Ministry of Health and Child Welfare, PMTCT Programme 2008 Annual Report, AIDS \& TB Unit, 2008.

[6] Ministry of Health and Child Welfare, Module 9: More Efficacious ARV Prophylaxis of Mother to Child Transmission (PMTCT), 2008.
[7] United Nations General Assembly Special Session, Zimbabwe Country Report on HIV and AIDS Reporting Period: January 2008 to December 2009, 2010.

[8] N. Oberzaucher and R. Baggaley, HIV Voluntary Counselling and Testing: A Gateway to Prevention and Care, 2002.

[9] Ministry of Health and Child Welfare, Towards Universal Access to HIV and AIDS Prevention, Treatment, Care and Support; The National HIV Testing and Strategic Plan 20082010, 2008.

[10] Southern African Regional Network for Equity in Health (EQUINET), ART Treatment Access and Effective Responses to HIV and AIDS-Providing new Momentum for Accessible, Effective and Sustainable Health Systems: Issues and Options Briefing December 2003, 2003.

[11] S. Ray and T. Kureya, Zimbabwe's Challenge: Equity in Health Sector Responses to HIV and AIDS in Zimbabwe, EQUINET Discussion Paper Number 9, August 2003, 2003.

[12] W. M. El-Sadr, M. Rabkin, R. Atun, and K. M. De Cock, "Bridging the divide," Journal of Acquired Immune Deficiency Syndrome, vol. 57, supplement 2, 2011.

[13] ART Data Verification \& Cohort Analysis in 22 districts, Global Fund Round 5 Zimbabwe (1-6 November, 2009).

[14] R. Atun and J. Bataringaya, "Building a durable response to HIV/AIDS: implications for health systems," Journal Acquired Immune Deficiency Syndrome, vol. 57, pp. S91-S95, 2011.

[15] Y. M. Dambisya, S. I. Modipa, and N. Z. Nyazema, A Review on the Impact of HIV and Programmes on Health Worker Retention, Norman Health Systems Research Group, Department of Pharmacy, University of Limpopo, Mankweng, South Africa, 2009.

[16] R. Levine and N. Oomman, "Global HIV/AIDS funding and health systems: Searching for the win-win," Journal of Acquired Immune Deficiency Syndromes, vol. 52, supplement 1, pp. S3S5, 2009.

[17] UNAIDS, Voluntary Counselling and Testing (VCT), Technical Update, Geneva, Switzerland, 2000.

[18] D. L. Kodner and C. Spreeuwenberg, "Integrated care: meaning, logic, applications, and implications," A Discussion Paper: International, 2002.

[19] D. K. Ekouevi, S. Karcher, and P. A. Coffie, "Strengthening health systems through HIV monitoring and evaluation in sub-saharan Africa," Current Opinion in HIV \& AIDS, vol. 6, no. 4, pp. 245-250, 2011.

[20] D. S. Jones, M. Tshimanga, G. Woelk et al., "Increasing leadership capacity for HIV/AIDS programmes by strengthening public health epidemiology and management training in Zimbabwe," Human Resources for Health, vol. 7, article 69, 2009.

[21] D. Yu, Y. Souteyrand, A. Mazuwa, M. A. Banda, J. Kaufman, and J. H. Perriëns, "Investment in HIV/AIDS programs: does it help strengthen health systems in developing countries?" Globalization and Health, vol. 4, article 8, 2008. 


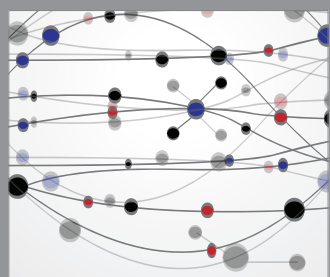

The Scientific World Journal
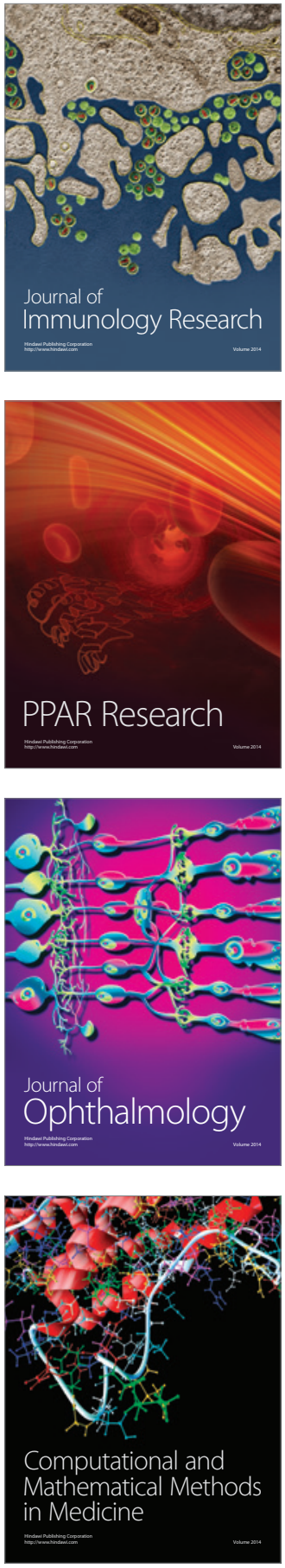

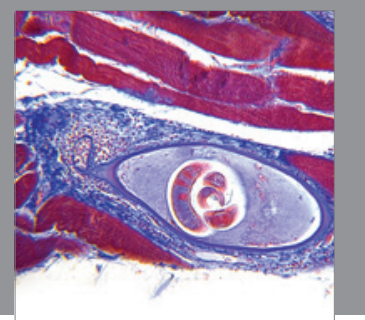

Gastroenterology

Research and Practice
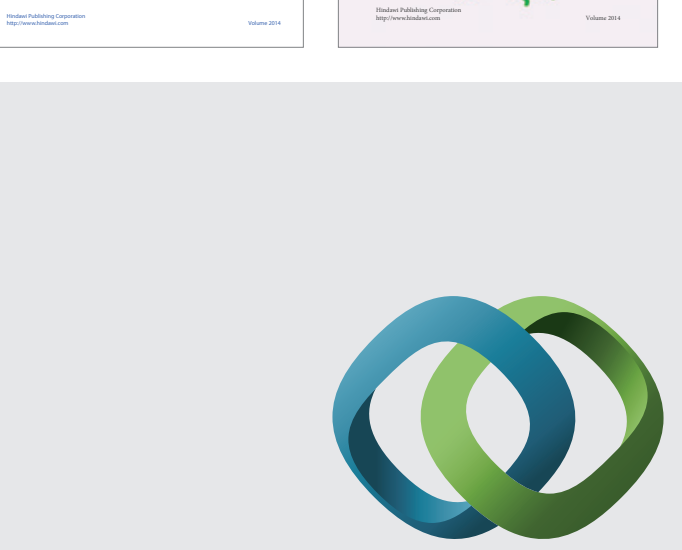

\section{Hindawi}

Submit your manuscripts at

http://www.hindawi.com
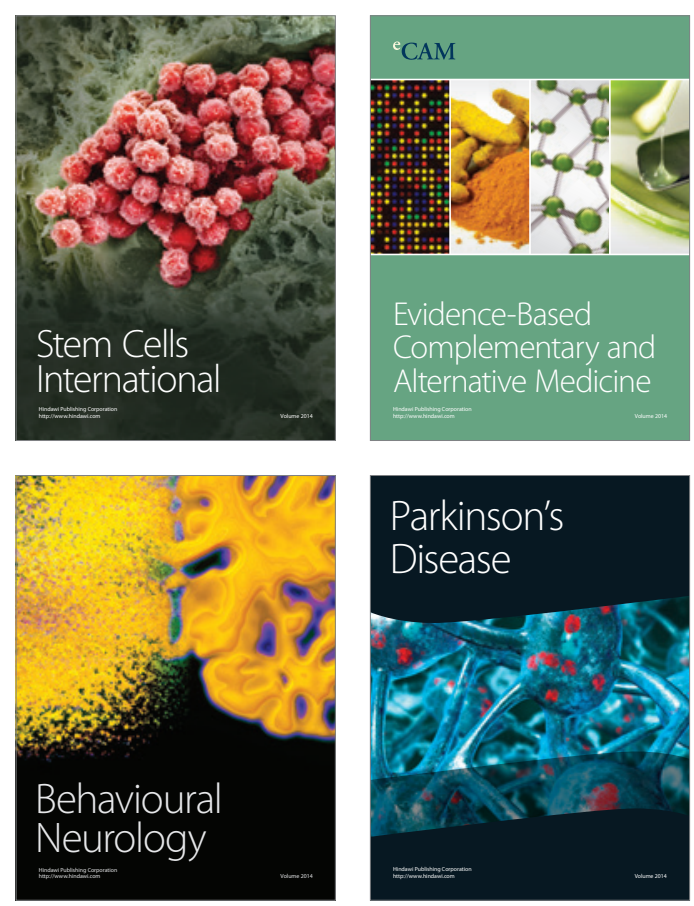

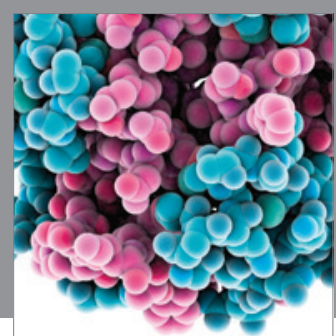

Journal of
Diabetes Research

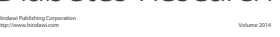



Disease Markers
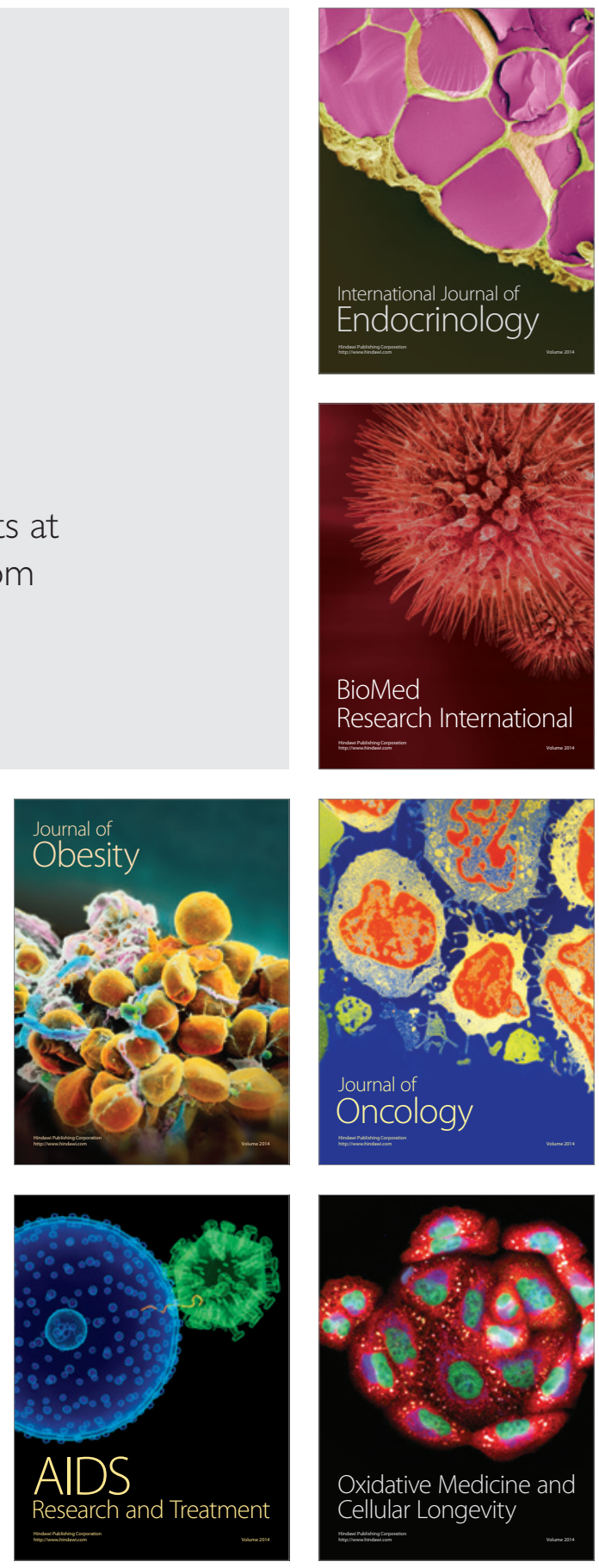